\title{
ENTORNO DE ALTA INCERTIDUMBRE PARA LA EMPRESA EN EL PERÚ: EL CASO DE EXPLORACIÓN Y PRODUCCIÓN DE HIDROCARBUROS EN EL SECTOR PRIVADO
}

\section{ENVIRONMENT OF HIGH UNCERTAINTY FOR THE COMPANY IN PERU: THE CASE OF HYDROCARBON EXPLORATION AND PRODUCTION IN THE PRIVATE SECTOR}

\author{
Gianine Tejada Salinas
}

RECEPCIÓN: 04 DE OCTUBRE DE 2021

ACEPTACIÓN: 05 DE NOVIEMBRE DE 2021

\section{RESUMEN}

Actualmente, las empresas enfrentan un ambiente competitivo. La producción de nuevos materiales y tecnologías y la crisis causada por la pandemia global por Covid-19 en la que la industria, la minería, los hidrocarburos y las universidades están inmersos plantean nuevos retos para enfrentar el futuro de una sociedad que exige de las empresas públicas y privadas, de la educación y de la sociedad en general, compromisos para la innovación, la investigación y el desarrollo sostenible de sus actividades y el entorno de los ecosistemas. En este trabajo, se presenta algunos aspectos de la situación de los hidrocarburos a nivel mundial, nacional y su evolución para la seguridad energética del Perú. Asimismo, busca examinar las variables relacionadas al macroambiente de la industria petrolera y pretende determinar las oportunidades que podrían beneficiar a la empresa y las amenazas que se deben eludir. Se toman aspectos del 2007 como punto de partida para posteriormente seguir indagando nuevos periodos. Del mismo modo, se evaluará cómo han cambiado los análisis proyectados en el período 2007-2021 y cómo la incertidumbre influye en dichos escenarios.

Palabras clave: Empresa, tendencias, innovación, complejidad, incertidumbre

\begin{abstract}
Today, companies face a competitive environment. The production of new materials and technologies and the crisis caused by the global pandemic by Covid-19 in which the industry, mining, hydrocarbons and universities are immersed, poses new challenges to face the future of a society that demands from public and private companies, education and society in general, commitments in innovation, research and sustainable development of their activities and the environment of ecosystems. In this paper, some aspects of the situation of hydrocarbons at the world and national level and their evolution for the energy security of Peru are presented. Likewise, it seeks to examine the variables related to the macroenvironment of the oil industry and seeks to determine the opportunities that could benefit the company and the threats that must be avoided. Aspects of 2007 are taken as a starting point to later continue investigating new periods. In the same way, it will be evaluated how the projected analyzes have changed in the 2007-2021 period and how uncertainty influences these scenarios.
\end{abstract}

Keywords: Company, trends, innovation complexity, uncertainty 


\section{INTRODUCCIÓN}

Las empresas están tomando cada vez más conciencia de la importancia del planeamiento estratégico en la dirección empresarial, pero eso no es suficiente. El surgimiento de la IV revolución industrial(como producto de los desarrollos en la biología molecular y la mecánica cuántica), la dinámica compleja de los sistemas alejados del equilibrio, la alta incertidumbre de los precios y variables, así como de los procesos externos e internos, demuestran que se requiere de un conocimiento anticipado de los riesgos y desafíos, de una visión prospectiva para la creación de escenarios posibles, la toma de decisiones y las políticas públicas del presente. Las empresas requieren de nuevos marcos epistémicos y otras formas de pensar y dirigir para enfrentar un ambiente competitivo, que se caracteriza por la producción de nuevos materiales, la pandemia global que ha afectado a la industria, la minería, los hidrocarburos, y a las propias universidades; así como los nuevos retos y compromisos que la sociedad exige de las empresas públicas, privadas, de la educación, entre otros sectores para el desarrollo sostenido de sus actividades.

El presente trabajo plantea algunas de las variables relacionadas al macroambiente de la industria petrolera y busca determinar las oportunidades que podrían beneficiar a la empresa y las amenazas que se deben eludir. Asimismo, intenta evaluar los modos en que han cambiado los análisis proyectados para el período 2007- 2021 y la manera en que la incertidumbre influye en los escenarios. Para el examen del macroambiente, se utilizó el análisis del entorno general, el que ha permitido conocer el escenario en el cual se desenvuelven las empresas petroleras. Así, se centrará en las variables más importantes que afectan tanto a la empresa como a sus clientes, como las económicas, político-legales, tecnológicas, sociales y ecológicas tomando como punto de partida en este trabajo el ańo 2007.

\section{VARIABLE ECONÓMICA}

\subsection{La situación energética en el mundo (13)}

El consumo de petróleo se ha incrementado notablemente y seguirá incrementándose. En 1973, el consumo anual era de 2,753 MMBL (millones de barriles) y para el 2004 llegó a 3,767 MMBL. La extracción diaria de petróleo es de unos $75 \mathrm{MMBL}$ y se espera que la demanda se incremente $2 \%$ por año, por lo que para el 2020 se necesitará unos $100 \mathrm{MMBL}$ diarios. Actualmente, se augura un crecimiento energético del $50 \%$ en los próximos 15 años.

La seguridad energética hoy en día es un tema fundamental, lo que significa que es necesario asegurar de fuentes energéticas a precios que permitan un adecuado crecimiento de la economía. Esto se puede hacer mediante políticas de cooperación o conflicto. El primero será factible en la medida que el recurso energético sea abundante; mientras que el segundo, en cambio, se irá acentuando conforme el recurso se vaya haciendo más escaso. Esto último es precisamente lo que esta ocurriendo.

América Latina tiene una ventaja a considerar en el tema de energía en relación con otros países dl globo. La región es inmensamente rica en reservas de petróleo, gas natural, carbón mineral, potenciales eólico, solar e hidroeléctricos. De acuerdo con cálculos conservadores, se estima que las reservas de gas y petróleo en América Latina pueden alcanzar para un periodo de 40 años. Las reservas de petróleo recién descubiertas en Brasil han sido consideradas como el hallazgo más grande e importante del hemisferio occidental en los últimos 30 años. Se estima que las reservas petroleras brasileñas aumentarán en un $50 \%$ y convertirá al país en la octava nación con mayores reservas de petróleo y gas del mundo. 
Los hidrocarburos líquidos han logrado importancia en el panorama energético mundial por la explotación de grandes reservas no convencionales de petróleo y gas. La caída del precio internacional del petróleo tiene como una de sus principales causas el exceso de oferta proveniente del shale oil y shale gas (petróleo y gas de esquisto) en Estados Unidos, y que la Organización de Países Exportadores de Petróleo (OPEP) decidió no reducir su cuota de producción para mantener su participación de mercado ante la amenaza de la entrada de nuevas fuentes de oferta no convencional.

El panorama de la energía mundial se encuentra en un proceso de transformación, debido a varios factores. Cabe mencionar los costos cada vez más bajos de la energía limpia, las tecnologías disruptivas, las redes inteligentes, los medidores inteligentes y los sistemas de datos geoespaciales han transformado la planificación energética. En este sentido, el Dr. Fatih Birol, director ejecutivo de la Agencia Internacional de Energía (AIE) señaló que: “... hacia el logro de cero emisiones netas para 2050, podemos alcanzar metas claves en materia de energía sostenible para 2030, a medida que ampliemos el acceso a las energías renovables en todos los sectores y aumentemos la eficiencia energética”. (1)

\subsection{La situación energética en el Perú (20)}

La situación energética en el mundo en las últimas décadas enfrenta muchas incertidumbres que se reflejan en el panorama nacional. Cabe preguntarnos ¿cuál es el panorama de la seguridad energética en el Perú?, ¿`cuál es el rol de los hidrocarburos en la economía?.

Durante el año 2007, se suscribieron 24 nuevos contratos de licencia para la exploración y explotación de hidrocarburos, y se contaron un total de 84 contratos (19 en exploración y 65 en explotación) dicho año. Estos nuevos contratos representan una inversión proyectada de US\$ 809 MM (millones). El nivel de inversiones en las actividades de exploración y explotación en dicho año fue del orden de US\$ $960 \mathrm{MMl}$, lo que significó un incremento del 39.7\% en relación con el año 2006, en el que se registró una inversión de US\$ 687 MM. En octubre del 2007, se perforaron 8 pozos exploratorios y 139 pozos de desarrollo. De estos últimos, 130 están ubicados en el noroeste del país, 7 en la selva y 2 en el zócalo continental.

Según el Plan Referencial de Hidrocarburos 2007-2016 (11), se estima que para el 2016 la demanda total de hidrocarburos (que incluye el gas natural) irá creciendo a una tasa de 6,3\% anual. La producción nacional de hidrocarburos líquidos será de 115,9 MBPD (miles de barriles por día) en el ańo 2007 y de 251,7 MBPD en el ańo 2016.

Para el año 2008, se esperaba que el sector se recupere considerablemente al expandirse $6.6 \%$ en virtud del crecimiento del subsector minero metálico (6.3\%), explicado por la creciente producción de cobre, zinc y plata. A esto se suma la evolución favorable en la producción de hidrocarburos (10\%) como resultado de la exploración y explotación de gas natural. Respecto de las exportaciones del sector, estas se incrementarían en $6 \%$, producto de mayores envíos de minerales metálicos $(6.3 \%)$ y al aumento de las ventas al exterior de hidrocarburos (3.9\%).

El proyecto Camisea (agosto de 2004) y la promulgación de un marco regulatorio promotor de la industria del gas natural marcaron un hito histórico que ha producido la transformación de la matriz energética primaria del país, en particular del sector eléctrico. Esto ha mejorado la balanza comercial de hidrocarburos y permitido que los hogares peruanos, la industria, el comercio y el transporte se beneficien de un combustible amigable con el medio ambiente y de bajo costo. 
TaBla 1. Análisis de tendencias económicas

\begin{tabular}{|c|c|c|}
\hline TENDENCIA 2007 & CLIENTES & NEGOCIO \\
\hline $\begin{array}{l}\text { Se estima tener reservas petroleras hasta el } 2030 \text {. Antes, por cada } \\
2.5 \text { barriles de petróleo consumido se descubría } 1 \text {, ahora por cada } \\
5 \text { solo } 1 \text {. }\end{array}$ & $\begin{array}{l}\text { FAVORABLE: } \\
\text { Oportunidad para } \\
\text { inversión en estudios } \\
\text { de exploración y } \\
\text { explotación. }\end{array}$ & \multirow{12}{*}{$\begin{array}{l}\text { FAVORABLE: } \\
\text { Mayores } \\
\text { consultorías, } \\
\text { desarrollo } \\
\text { de alianzas } \\
\text { con empresas } \\
\text { internacionales, } \\
\text { desarrollo de } \\
\text { nuevos servicios }\end{array}$} \\
\hline $\begin{array}{l}\text { Fin del petróleo barato por la creciente demanda en países } \\
\text { asiáticos y conflictividad en medio oriente. }\end{array}$ & \multirow{3}{*}{$\begin{array}{l}\text { FAVORABLE: } \\
\text { Elevación de precios } \\
\text { del petróleo, que } \\
\text { llegó a cotizarse en } \\
\text { marzo } 2008 \text { US \$ } \\
110,20 .\end{array}$} & \\
\hline $\begin{array}{l}\text { Incremento del precio de petróleo debido a factores como la } \\
\text { disminución de tasa de interés EEUU, debilidad del dólar } \\
\text { americano, incertidumbre del abastecimiento del crudo, guerras } \\
\text { internacionales, incremento de demanda de China e India. }\end{array}$ & & \\
\hline $\begin{array}{l}\text { Incremento del consumo petróleo, crecimiento energético del 50\% } \\
\text { en los próximos } 15 \text { ańos. }\end{array}$ & & \\
\hline $\begin{array}{l}\text { América Latina es rica en reservas de hidrocarburos, especialmente } \\
\text { en crudos pesados. }\end{array}$ & $\begin{array}{l}\text { FAVORABLE: } \\
\text { Mayores inversiones. }\end{array}$ & \\
\hline $\begin{array}{l}\text { Impulso del reemplazo de gasolina por biodiesel y bioetanol en } \\
\text { base a vegetales (maíz, caña de azúcar, palma africana) por Brasil, } \\
\text { Colombia, Argentina, EEUU, India, Tailandia, China, Unión } \\
\text { Europea. }\end{array}$ & \multirow{2}{*}{$\begin{array}{l}\text { FAVORABLE: } \\
\text { Diversificación de sus } \\
\text { negocios. }\end{array}$} & \\
\hline $\begin{array}{l}\text { El carbón jugará un papel importante por medio de centrales } \\
\text { térmicas a carbón limpio que tienen baja emisión de dióxido de } \\
\text { carbono. }\end{array}$ & & \\
\hline $\begin{array}{l}\text { Incremento de inversiones en actividades de exploración y } \\
\text { explotación. }\end{array}$ & \multirow{5}{*}{$\begin{array}{l}\text { FAVORABLE: } \\
\text { Incremento de } \\
\text { actividad exploratoria } \\
\text { en hidrocarburos en } \\
\text { el país. }\end{array}$} & \\
\hline $\begin{array}{l}\text { Demanda de hidrocarburos líquidos afectada por consumo de } \\
\text { gas natural e incremento de actividad económica del país (Plan } \\
\text { referencial de hidrocarburos 2007-2016). (11) }\end{array}$ & & \\
\hline $\begin{array}{l}\text { Acciones de PERÚPETRO y MEM dirigidas a activar la actividad } \\
\text { exploratoria para encontrar nuevas reservas. }\end{array}$ & & \\
\hline $\begin{array}{l}\text { El Estado a través de PERÚPETRO recibe regalías a consecuencia } \\
\text { de la actividad extractiva de hidrocarburos (canon y sobrecanon). }\end{array}$ & & \\
\hline $\begin{array}{l}\text { Se espera que la balanza comercial de hidrocarburos se vuelva } \\
\text { positiva por el inicio de operaciones del proyecto de exportación } \\
\text { de gas. }\end{array}$ & & \\
\hline
\end{tabular}

Elaboración propia

\subsection{Variable político y legal (20)}

PERÚPETRO ha modificado el modelo de contrato de licencia (17) que ha otorgado ciertos beneficios:

- La flexibilización del programa mínimo de trabajo exploratorio

- La incorporación de reevaluación luego de la perforación de los pozos exploratorios no exitosos

- La ampliación de la fase de exploración de siete a diez años 
- La exoneración del IGV, del impuesto de promoción municipal y de cualquier otro impuesto por un período de 5 años

- Convenio de estabilidad tributaria para el pago del impuesto a la renta

De acuerdo con la tabla 2, las tendencias político-legales son parcialmente favorables para el negocio ya que, pese a existir algunos beneficios en los contratos, estos se ven reducidos por la inestabilidad de ciertos factores como la corrupción, los conflictos sociales y los tiempos demasiado extensos para la aprobación de estudios de impacto ambiental.

TABLA 2. Análisis de tendencias político y legal

\begin{tabular}{|c|c|c|}
\hline TENDENCIA & CLIENTES & NEGOCIO \\
\hline Carencia de política energética debidamente orientada. & \multirow[b]{2}{*}{$\begin{array}{l}\text { DESFAVORABLE: } \\
\text { Incertidumbre por } \\
\text { carencia de políticas claras }\end{array}$} & \multirow[b]{2}{*}{$\begin{array}{l}\text { DESFAVORABLE: } \\
\text { Falta de políticas } \\
\text { claras }\end{array}$} \\
\hline $\begin{array}{l}\text { Factor relevante es la inestabilidad política del país: } \\
\text { corrupción, polarización de la sociedad, conflictos sociales. } \\
\text { El factor riesgo país está asociado a tres factores que son } \\
\text { riesgo del sistema financiero, riesgo default (no pago de la } \\
\text { deuda) y el tipo de cambio (nominal y esperado). }\end{array}$ & & \\
\hline $\begin{array}{l}\text { Beneficios en contrato de licencia por PERÚPETRO a } \\
\text { fin de incentivar y promover inversiones en exploración y } \\
\text { explotación de hidrocarburos; lograr que descubrimientos } \\
\text { menores, que no sean viables económicamente con la } \\
\text { estructura vigente, puedan serlo con la aplicación del } \\
\text { descuento referido; mejorar los niveles de rentabilidad de } \\
\text { los proyectos. }\end{array}$ & $\begin{array}{l}\text { FAVORABLE: Mejora de } \\
\text { rentabilidad de proyectos. }\end{array}$ & $\begin{array}{l}\text { FAVORABLE: } \\
\text { Ejecución de } \\
\text { mayores proyectos. }\end{array}$ \\
\hline $\begin{array}{l}\text { Obligatoriedad de realizar estudios de impacto ambiental } \\
\text { para inicio de actividades de exploración y explotación } \\
\text { de hidrocarburos, las mismas que tienen una duración } \\
\text { de } 2 \text { ańos. Este período es demasiado extenso comparado } \\
\text { con otros países de la región. }\end{array}$ & $\begin{array}{l}\text { DESFAVORABLE: } \\
\text { Demora en la aprobación } \\
\text { de proyectos que no } \\
\text { permite aprovechar la } \\
\text { bonanza del precio del } \\
\text { petróleo. }\end{array}$ & $\begin{array}{l}\text { DESFAVORABLE: } \\
\text { Menor oportunidad } \\
\text { de ejecución de } \\
\text { proyectos. }\end{array}$ \\
\hline
\end{tabular}

Elaboración propia

\subsection{Variable tecnológica (11)}

Las empresas que realizan exploración deben realizar inversiones específicas para la adquisición y mantenimiento de equipos exploratorios de alta tecnología, captación de profesionales y búsqueda de consultores de alta especialización a fin de poder minimizar sus riesgos. Para el caso de explotación existe una reducida inversión en nuevas tecnologías de extracción. De acuerdo con la tabla 3, resulta favorable para el negocio por la tecnología con la que cuentan las empresas nacionales.

TABLA 3. Análisis de tendencias tecnológicas

\begin{tabular}{|l|l|l|}
\hline \multicolumn{1}{|c|}{ TENDENCIA } & \multicolumn{1}{|c|}{ CLIENTES } & \multicolumn{1}{c|}{ NEGOCIO } \\
\hline Inversiones para adquisición y mantenimiento de equipos & $\begin{array}{l}\text { DESFAVORABLE: } \\
\text { Necesidad de adquirir y } \\
\text { contratar nuevos servicios }\end{array}$ & $\begin{array}{l}\text { FAVORABLE: } \\
\text { Mayor oportunidad } \\
\text { de negocios }\end{array}$ \\
\hline $\begin{array}{l}\text { Desarrollo de nuevos servicios acorde a avances } \\
\text { tecnológicos }\end{array}$ &
\end{tabular}

Elaboración propia 


\subsection{Variable social}

El gobierno derogó mediante los decretos leyes 977 y 978, expedidos el 15 de marzo de 2007, la mayor cantidad de exoneraciones tributarias que gozaban las regiones de la Amazonía gracias a la ley 27037 (21). Con la promulgación de los referidos decretos leyes, se derogaron las exoneraciones del impuesto a la renta en cuanto a crédito tributario por reinversión -devolución del Impuesto General a las Ventas (IGV) por compra de bienes-, así como la exoneración del IGV a la venta de bienes y prestación de servicios y contratos de construcción. Además, se elimina la exoneración del Impuesto Selectivo al Consumo (ISC) a los combustibles en las regiones de Loreto, Ucayali y Madre de Dios. Según los cálculos del Ministerio de Economía y Finanzas las exoneraciones amazónicas representaban el 0.4 del PBI (S/. 926 millones). Estas tendencias sociales afectan de igual forma tanto a los clientes como al negocio según lo mostrado en la tabla 4.

TABLA 4. Análisis de tendencias sociales

\begin{tabular}{|l|l|l|}
\hline \multicolumn{1}{|c|}{ TENDENCIA } & \multicolumn{1}{|c|}{ CLIENTES } & \multicolumn{1}{c|}{ NEGOCIO } \\
\hline Aportes al canon minero y voluntario para el desarrollo local. & $\begin{array}{l}\text { FAVORABLE: Mayores facilidades } \\
\text { para la empresa. }\end{array}$ & $\begin{array}{l}\text { FAVORABLE: Mayores } \\
\text { facilidades }\end{array}$ \\
\hline $\begin{array}{l}\text { Las actividades mineras y de hidrocarburos promueven un empleo } \\
\text { descentralizado y multiplicador. }\end{array}$ & $\begin{array}{l}\text { FAVORABLE: Empleo de mano de } \\
\text { obra local a bajo costo. }\end{array}$ & $\begin{array}{l}\text { FAVORABLE: Mano de } \\
\text { obra barata }\end{array}$ \\
\hline Descontento de la población por no sentir el crecimiento económico. & $\begin{array}{l}\text { DESFAVORABLE: Resentimiento a } \\
\text { empresas de hidrocarburos. }\end{array}$ & $\begin{array}{l}\text { DESFAVORABLE: } \\
\text { Resentimiento a } \\
\text { empresas. }\end{array}$ \\
\hline Derogación de exoneraciones tributarias en amazonia. & $\begin{array}{l}\text { DESFAVORABLE: Incertidumbre por } \\
\text { políticas del gobierno. }\end{array}$ & $\begin{array}{l}\text { DESFAVORABLE: } \\
\text { Incertidumbre por } \\
\text { políticas del gobierno. }\end{array}$ \\
\hline
\end{tabular}

Elaboración propia

\subsection{Variable ecológica}

No se puede realizar ningún trabajo de exploración sin antes haber realizado los estudios de impacto ambiental y los talleres de presentación del proyecto a las comunidades involucradas. El desarrollo de las actividades mineras y de hidrocarburos provoca insatisfacción en un sector de la población, debido a una fuerte corriente que se opone al desarrollo de las mismas, principalmente, por los perjuicios contra la naturaleza.

A nivel internacional se han realizado varias cumbres como la realizada en Brasil en el año 1992, denominada "Cumbre de la Tierra", y el Protocolo de Kyoto desarrollado en Japón. Además existen diferentes certificaciones internacionales que impulsan la conservación del medio ambiente.

TABLA 5. Análisis de tendencias ecológicas

\begin{tabular}{|c|c|c|}
\hline TENDENCIA & CLIENTES & NEGOCIO \\
\hline $\begin{array}{l}\text { Tendencia a la protección mundial del medio ambiente por } \\
\text { organismos internacionales y locales. }\end{array}$ & \multirow{4}{*}{$\begin{array}{l}\text { DESFAVORABLE: } \\
\text { Demora en la } \\
\text { aprobación de los } \\
\text { proyectos. }\end{array}$} & \multirow{4}{*}{$\begin{array}{l}\text { DESFAVORABLE: } \\
\text { Menor oportunidad } \\
\text { de realizar proyectos. }\end{array}$} \\
\hline $\begin{array}{l}\text { Fuerte presencia de organismos internacionales y locales } \\
\text { destinados a la protección del medio ambiente. }\end{array}$ & & \\
\hline Sanciones por dańos ecológicos muy severos. & & \\
\hline Población insatisfecha por algunos daños a la naturaleza. & & \\
\hline
\end{tabular}

Elaboración propia 
Estas eran algunas de las tendencias registradas en el año 2007 y duraron más o menos con fluctuaciones hasta el año 2015, cuando empieza la crisis internacional debido a la emergencia de nuevos patrones de comportamiento en la explotación petrolera especialmente en Estados Unidos debido a la explotación del petróleo.

La inversión en el sector hidrocarburos se ha reducido significativamente en los últimos años. En el período 2007-2021, la reducción de la inversión en el sector fue del 77.74\%.

Figura 1. Inversión en el Sector Hidrocarburos

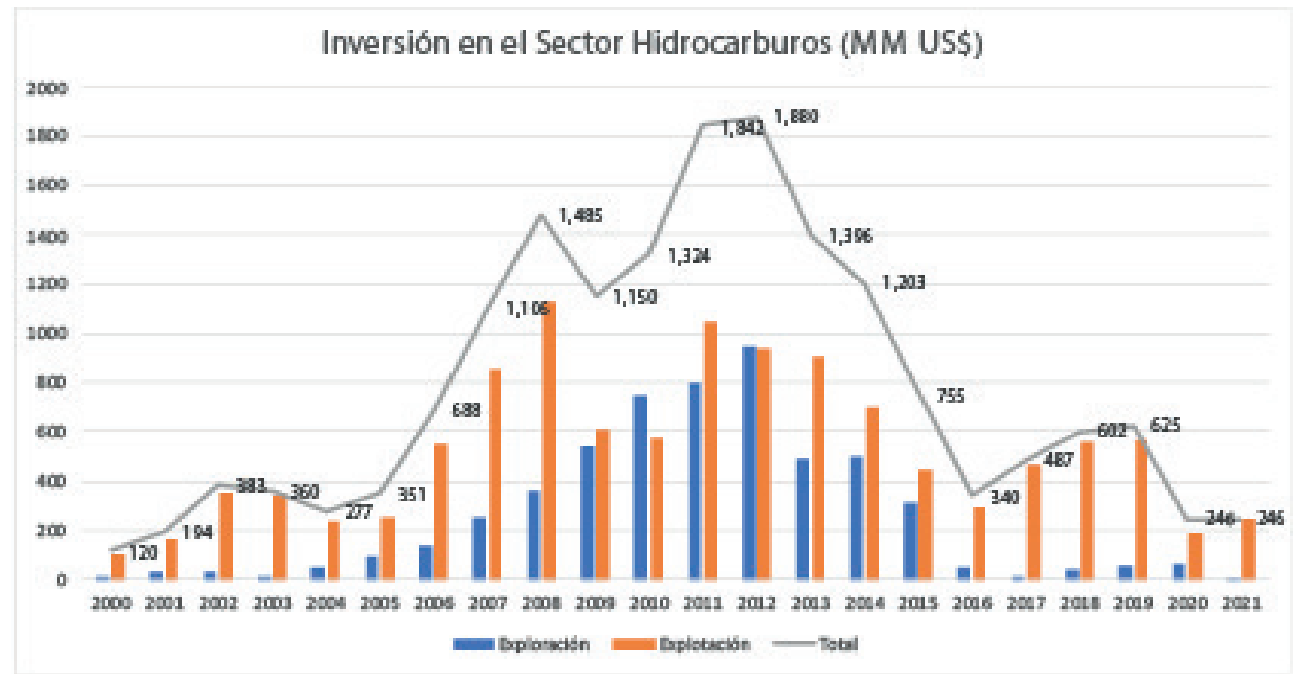

Fuente: Perupetro, información a noviembre de 2021. Elaboración Propia.

Figura 2. Contratos de Exploración y Explotación de Hidrocarburos suscritos, vigentes y terminados

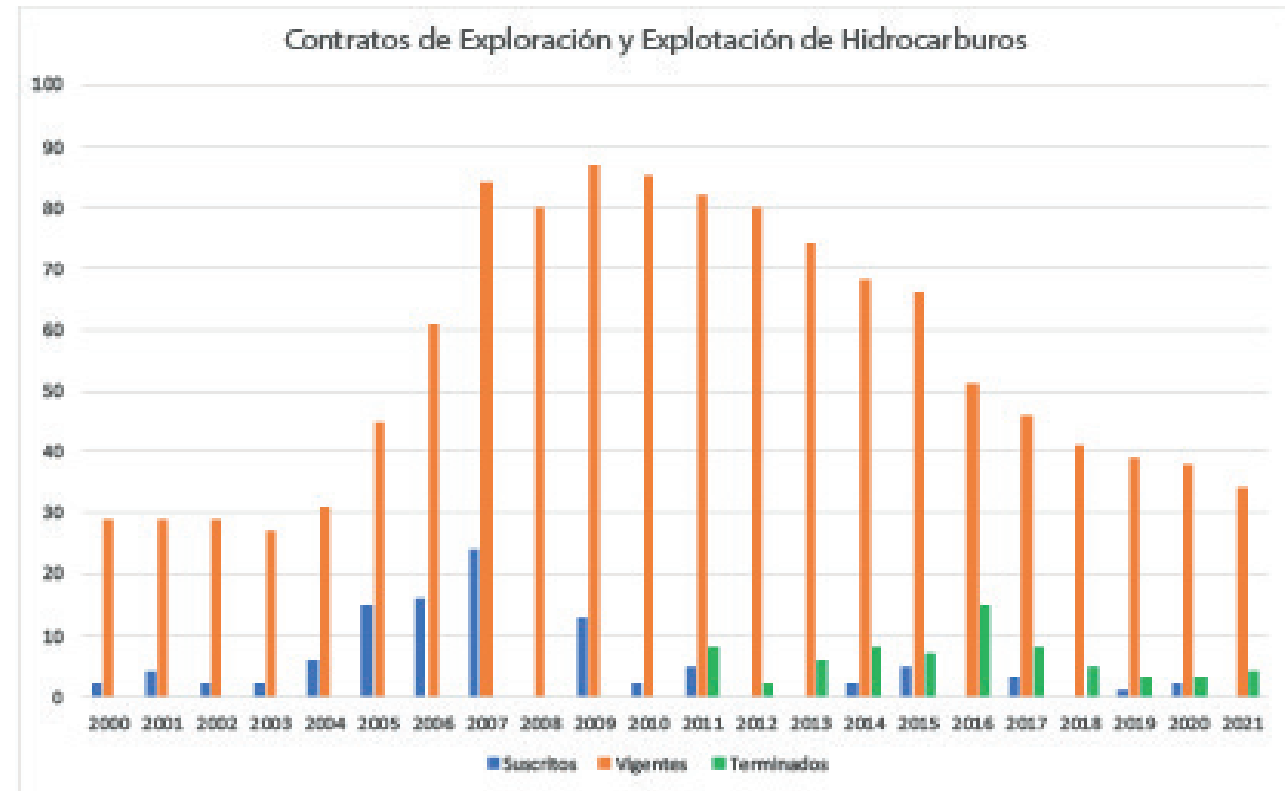

Fuente: Perupetro, información a noviembre de 2021. Elaboración: Propia. 
En la Figura 2, se aprecia que los contratos de exploración en el período 2007-2013 se encuentran en un mejor nivel debido al boom del precio del petróleo, mientras que a partir del 2013 empieza su declive. Esta situación pone en riesgo la seguridad energética del país. Gustavo Navarro, ex titular de la Dirección General de Hidrocarburos (DGH) señaló que en la actualidad, la producción nacional de petróleo es de 40 mil barriles diarios, aproximadamente, es decir, se necesita importar el 80\%, aproximadamente, de los combustibles que se consume en el país. Perú consume más de 250 mil barriles diarios de combustibles, sin incluir el gas natural. Petroperú está importando productos terminados como gasolinas, diesel, turbo. La Pampilla importa petróleo que refina en sus instalaciones. (21)

En momentos en que la producción petrolera sigue cayendo en el país al igual que las inversiones en hidrocarburos, uno de los incentivos más reclamados por las empresas del ramo es la reducción de regalías para incentivar mayor inyección de capital privado en esa actividad. Sin embargo, lejos de recoger esos requerimientos, el Ministerio de Energía y Minas (MINEM) evalúa ahora un proyecto de modificación del reglamento de regalías que le presentó la agencia Perupetro, y que plantea elevar las tasas de las regalías vigentes. En el Perú el rango promedio de las regalías que pagan las empresas petroleras oscila entre $5 \%$ y $25 \%$, pero en la referida propuesta, según el director gerente de Enerconsult, Carlos Gonzales, Perupetro está proponiendo elevar esa regalía hasta un 40\%.

\section{CONCLUSIONES}

- A nivel macroambiente, desde 2007 hasta el 2013, las perspectivas de crecimiento del negocio de exploración y explotación de hidrocarburos eran favorables para el desarrollo de consultoría. A partir de ahí, se percibe la tendencia a desaparecer los estudios de exploración, con lo cual han entrado en crisis las compañías nacionales que se dedicaban al estudio de exploración. De esta manera, el país pierde infraestructura, plataformas tecnológicas, capital humano especializado y gestión del conocimiento de la geología de hidrocarburos del territorio nacional.

- Para continuar en esa línea, se necesitan políticas claras del gobierno actual y de los futuros que alienten la inversión en este sector.

- Se requieren hacer investigaciones sobre el desempeño de las empresas nacionales y la problemática que enfrentan, que incluye la falta de apoyo del Estado, la competencia con subsidiaras de las grandes compañías petroleras que solo quieren tercerizar el trabajo y las amenazas de la corrupción.

- Se requiere evaluar la capacidad de inversión de compañías extranjeras que asumen contratos de exploración y hacer un seguimiento de si realmente invierten o solo buscan transferir los campos petroleros y establecer negocios que las beneficien pero sin asegurar el desarrollo de la exploración y explotación de los hidrocarburos en el Perú.

- Se requiere hacer estudios de futuro que permitan la construcción de escenarios para la toma de decisiones en materia energética en el Perú para lograr la seguridad energética y el bienestar social.

- Se requiere generar un ambiente de sostenibilidad en las comunidades nativas de las regiones en las que se están llevando tareas de exploración y producción. De esta manera, se asegura que participen de los beneficios de la producción petrolera. Esto les permitirá, a su vez, mejorar los niveles de educación, negocios sostenibles y cuidado del medio ambiente. 


\section{REFERENCIAS BIBLIOGRÁFICAS}

[1] Banco Mundial. (2021). El acceso universal a la energía sostenible seguirá siendo inalcanzable, a menos que se aborden las desigualdades. Disponible online: https://www.bancomundial.org/es/ news/press-release/2021/06/07/report-universal-access-to-sustainable-energy-will-remain-elusive-without-addressing-inequalities

[2] Benavides, C. y Quintana, C. (2003). Gestión del Conocimiento y Calidad Total, Madrid, España: Díaz de Santos.

[3] Bueno, E., Paz, M. Merino, C. y Castilla, J. (2006) "Dirección estratégica, desarrollo de la estrategia y análisis de casos", Madrid, Editorial Pirámide.

[4 Butteriss M. (2001), "Reinventando Recursos Humanos”, España, Ediciones Gestión.

[5] David, F. (2003). "Conceptos de Administración Estratégica”, México, Pearson Education, Novena edición.

[6] Grant, R., (1996) “Dirección Estratégica. Conceptos, Técnicas y Aplicaciones”, Madrid, Editorial Civitas.

[7] Kaplan R., Norton D. (2002) “Cuadro de Mando Integral”, Gestión 2000.com.

[8] Kaplan R., Norton D. (2004) "Mapas Estratégicos", Gestión 2000.com.

[9] Kotler, P. (2000), "Dirección de Marketing”, España, Editorial Pretince Hall, 2000.

[10] Ministerio de Energía y Minas. (2008). Disponible en: http://www.minem.gob.pe/hidrocarburos/pub_planreferen_2006.asp.

[11] Ministerio de Energía y Minas. (2008). "Diagnóstico situacional del sector Energía y Minas y de las Instituciones Públicas Descentralizadas al Congreso de la República"

[12] Navas J., Guerras M., (1998). "La Dirección Estratégica de la Empresa. Teoría y Aplicaciones", Madrid, Editorial Civitas.

[13] Obando, E. (2007). La energía como tema de seguridad en América del Sur. Centro de Altos Estudios Nacionales (CAEN).

[14] OSINERGMIN. La Industria de los Hidrocarburos Líquidos en el Perú. Disponible online: https://www.osinergmin.gob.pe/seccion/centro_documental/Institucional/Estudios_ Economicos/Libros/Libro-industria-hidrocarburos-liquidos-Peru.pdf

[15] OSINERGIM (2005).Oficina de estudios económicos, "La Organización Económica de la Industria de Hidrocarburos en el Perú: El segmento Upstream del Sector Petrolero”, Documento No 8.

[16] Payne A., (1996) La esencia de la mercadotecnia de servicios, Editorial Prentice Hall Hispanoamérica, S.A., Mexico.

[17] Peru Petro. Estadística Anual de Hidrocarburos. Disponible en: http://www.perupetro.com.pel beneficios-s.asp, 31 Enero 2008, 10:49.

[18] Porter, M. (1982), "Estrategia Competitiva”, México, Editorial C.E.C.S.A.

[19] Porter, M., (1987) "Ventaja competitiva. Creación y sostenimiento de un desempeño superior", México, Editorial C.E.C.S.A.

[20] Riesco M. (2006) “El Negocio es el Conocimiento”, Díaz de Santos.

[21] Sociedad Nacional de Minería, Petróleo y Energía. Disponible en: https:/www.snmpe.org.pe/ prensa/sintesis-de-noticias/hidrocarburos.html

[22] University of Chicago Graduate School of Business, INSEAD, University of Michigan Business School, Said Business School Oxford University; "Financial times mastering strategy, The complete MBA companion in strategy". Great Britain, Pearson Education Limited, 2000. 